\title{
Distance Dependence of the Mn-Mn Exchange Interaction in IV-VI Semimagnetic Semiconductors
}

\author{
A. Łusakowski and M. Górska \\ Institute of Physics, Polish Academy of Sciences \\ al. Lotników 32/46, 02-668 Warszawa, Poland
}

\begin{abstract}
In the present paper we calculate the exchange interaction between two manganese ions in IV-VI semiconductors with the rocksalt structure. The method of calculations is based on the fourth order perturbation theory with respect to hybridization between band states and localized $d$ orbitals of $\mathrm{Mn}$ ions. This hybridization is described by three Harrison integrals: $V_{p d \sigma}, V_{p d \pi}$, and $V_{s d \sigma}$. The band states of IV-VI semiconductor are obtained from the semiempirical tight binding model built from $s$ and $p$ orbitals of cations and anions. The resulting exchange term in the Hamiltonian is of the form $-\sum_{i, j=x y z} J_{i j} \hat{S}_{i}^{1} \hat{S}_{j}^{2}$, however nondiagonal terms of the exchange integral tensor $J_{i \neq j}$ are very small. The dependence of $J_{i j}$ on the Mn-Mn distance is non-monotonic. We also discuss the influence of the local crystal deformations on the exchange integral.
\end{abstract}

PACS numbers: 71.70.Gm, 75.30.Hx

The exchange interaction between magnetic ions has been studied more extensively in II-VI semimagnetic semiconductors (SMSC) which crystallize in the zinc-blende structure than in the IV-VI SMSC possessing the rocksalt structure. According to the model by Larson et al., based on the $s p-d$ hybridization, the exchange integral in the II-VI SMSC should decrease monotonously with increasing distance between $\mathrm{Mn}$ ions [1]. However, more recent experimental and theoretical results indicated that in Zn-based II-VI SMSC the exchange integral between fourth neighbours may be larger than between third neighbours $[2,3]$.

The nearest neighbour exchange interaction in IV-VI semiconductors has been considered by Górska and Anderson [4]. Their calculations were based on the Anderson superexchange mechanism between Mn ions via intervening anions' orbitals [5]. The distance dependence of the $\mathrm{Mn}-\mathrm{Mn}$ exchange integral in IV-VI 
SMSC has been calculated by Liu and Bastard [6], Dugaev and Litvinov (DL) [7], and the temperature corrections to DL results have been analysed by Rusin [8].

In the present paper we analyse the same problem. However, our approach differs from the previous ones in two aspects. First, in Ref. [6] the band energy spectra have been assumed to be parabolic in the vicinity of $L$ point of the Brillouin zone (BZ) and in Refs. [7] and [8] they have been calculated within the framework of the Dimmock model [9]. Such models are appropriate for description of states in the vicinities of $L$ points of BZ only. In our calculations we use semiempirical tight binding model proposed for IV-VI semiconductors by Kriechbaum et al. [10]. In this model the band states are built from the $p$ and $s$ orbitals of cations and anions with the interactions with first and second neighbours taken into account. Such an approach enables us to calculate the energies and the wave functions for the band states not only in the vicinities of the $L$ points but in the entire BZ. The knowledge of the spectrum in a wide area of BZ is important for calculations of the $\mathrm{Mn}-\mathrm{Mn}$ exchange integrals for small $\mathrm{Mn}-\mathrm{Mn}$ distances $\boldsymbol{R}$. This is related to the fact that formulae for the $\mathrm{Mn}-\mathrm{Mn}$ exchange integrals contain integrals over BZ with integrands containing oscillating factors $\exp (\mathrm{i} \boldsymbol{k} \boldsymbol{R})$. For $\boldsymbol{R} \rightarrow \infty$, the main contributions to the integrals come from the vicinity of the band extrema and we think that in this limit the results of Refs. [6-8] are correct.

The second important difference between the present paper and Refs. [6-8] is the form of the interaction between band states and Mn spins. While in Refs. [6-8] this interaction has been assumed in the form of a Kondo-like Hamiltonian, $H_{\text {int }}=$ $J_{s p-d} \boldsymbol{S}_{\mathrm{Mn}} \cdot \sigma$, with one $s p-d$ exchange coupling $J_{s p-d}$, in the present paper we start from a more basic level, i.e. from the hybridization $H^{\prime}$ between band states and the $d$ orbitals of Mn atom

$$
H^{\prime}=\sum_{L \sigma n k}\left(V_{L \sigma, n k} a_{L \sigma}^{\dagger} a_{n k}+V_{L \sigma, n k}^{*} a_{n k}^{\dagger} a_{L \sigma}\right)
$$

The operator $a_{L \sigma}^{\dagger}$ creates an electron on the $d$ shell of $\mathrm{Mn}$ ion in the state with orbital momentum $L$ and spin $\sigma$. The operator $a_{n k}^{\dagger}$ creates a band electron in the state characterized by the band index $n$ and the wave vector $k$. Because our band states are built from atomic $p$ and $s$ orbitals the hybridization between Mn orbital $L \sigma$ and the band state $n \boldsymbol{k}$ described by $V_{L \sigma, n \boldsymbol{k}}$ may be expressed by three Harrison parameters [11]: $V_{s d \sigma}, V_{p d \pi}, V_{p d \sigma}$ known from the literature [12].

The method of calculations is based on the fourth order perturbation theory with respect to hybridization $V$ between band states and localized $d$ orbitals of Mn ions $[1,13]$. We assume that the valence band is completely filled with electrons and there are two possible excited states for a manganese ion: with six or four electrons on the $d$ shell. Applying formulae and methods similar to those in Refs. [1] and [13] we obtain the effective exchange Hamiltonian, $H_{\text {eff }}$, which may be expressed in the following form: 


$$
H_{\mathrm{eff}}(\boldsymbol{R})=\mathrm{const}-\sum_{i, j=x, y, z} J_{i j}(\boldsymbol{R}) \hat{S}_{i}^{1}(0) \hat{S}_{j}^{2}(\boldsymbol{R}),
$$

where $\hat{\boldsymbol{S}}^{1}$ and $\hat{\boldsymbol{S}}^{2}$ are the Mn ions spin operators. Each of the exchange integrals, $J_{i j}$, may be expressed as a sum of three terms: $J_{i j}=J_{i j}^{\mathrm{hh}}+J_{i j}^{\mathrm{he}}+J_{i j}^{\mathrm{ee}}$, where the superscripts correspond to virtual processes in perturbation calculations involving electrons from valence band only (hh), valence and conduction band (he), and conduction band (ee).

In general, in the real crystals the manganese ions are not in the perfect octahedral environment. Because the ionic radius of $\mathrm{Mn}$ is much smaller than the ionic radii of $\mathrm{Pb}$ or $\mathrm{Sn}$ and, moreover, the $\mathrm{Mn}$ atoms are distributed randomly in the lattice we expect that the lattice is locally deformed. In the ideal crystal $\mathrm{PbTe}$ or $\mathrm{SnTe}$, in the tight binding approximation, the band wave functions contain certain contributions from atomic orbitals of Te atoms. One of the main assumptions of the procedure is that the amplitudes of these orbitals do not change when $\mathrm{Pb}$ or Sn atom is replaced by manganese. We assume that in the limit of small Mn concentration these amplitudes are also not changed when the crystal lattice is locally deformed. With this assumption we may calculate hybridization elements $V_{L \sigma, n k}=\left\langle L \sigma\left|H^{\prime}\right| \chi_{n k}\right\rangle$ changing only the positions of tellurium atomic orbitals surrounding a manganese atom and leaving their amplitudes unchanged. From computer simulations we know that the deviations of the bonds' directions from their values in an ideal crystal with the Mn concentration up to $3 \%$ are of the order of $1^{\circ}-5^{\circ}[14]$. It turns out that the crystal lattice deformations lead to a decrease of about $1-3 \%$ in the absolute value of the exchange integral between the nearest neighbours and even less for the next neighbours.

The main results of our calculations are:

1. The non-Heisenberg terms in the Hamiltonian (2) are very small. $J_{i j}$ for $i \neq j$ are of the order of $0.001 \mathrm{~K}$.

2. For a given coordination zone the tensor components $J_{i j}$ practically do not depend on the crystallographic direction. For example $J_{x x}\left(\frac{a}{2}, \frac{a}{2}, 0\right)-$ $J_{x x}\left(0, \frac{a}{2}, \frac{a}{2}\right) \sim 0.001 \mathrm{~K}$. For a given $\boldsymbol{R}$ the differences between $J_{i i}$ and $J_{j j}$ for $i \neq j$ are also of the same order of magnitude. That means that the spin-spin interaction in IV-VI SMSC is well approximated by the Heisenberg Hamiltonian with one exchange integral, $J$, which is shown in Figs. 1 and 2. This is different from the results by Dugaev and Litvinov [7], where Heisenberg and non-Heisenberg terms are of the same order of magnitude.

3. The ratio of the next nearest to nearest neighbour exchange integral, $J_{\mathrm{NNN}} / J_{\mathrm{NN}}$, is substantially larger in IV-VI compounds than in II-VI compounds. We may understand this effect qualitatively if we notice that in the rocksalt structure starting from one cation site we must travel along two cation-anion bonds to reach the second nearest neighbour, while the shortest path connecting two NNN sites in the zinc-blende structure goes along 
four bonds. Thus, in this sense, the "distance" between NNN is much longer in II-VI than in IV-VI compounds.

4. The results for $\mathrm{PbTe}$ and $\mathrm{SnTe}$ for the same set of Harrison parameters are very similar (see Fig. 1b).

The above results lead to a conclusion that the interaction between manganese ions in IV-VI SMSC is in some sense "localized", i.e. it "propagates" mostly via orbitals of intervening anions and the details of the band structure are not crucial.

5. The contribution of $J_{\text {ee }}$ to $J$ is very small (see Fig. 1a). The contribution of $J_{\text {he }}$ is larger than in II-VI SMSC, however, this is not related to the crystallographic structure but rather to the smaller value of the energy gap.

6. The distance dependence of $J$ is non-monotonic (see Fig. 1) However, this effect appears for distances where the exchange integral is very small and it seems unlikely that it can be verified experimentally.

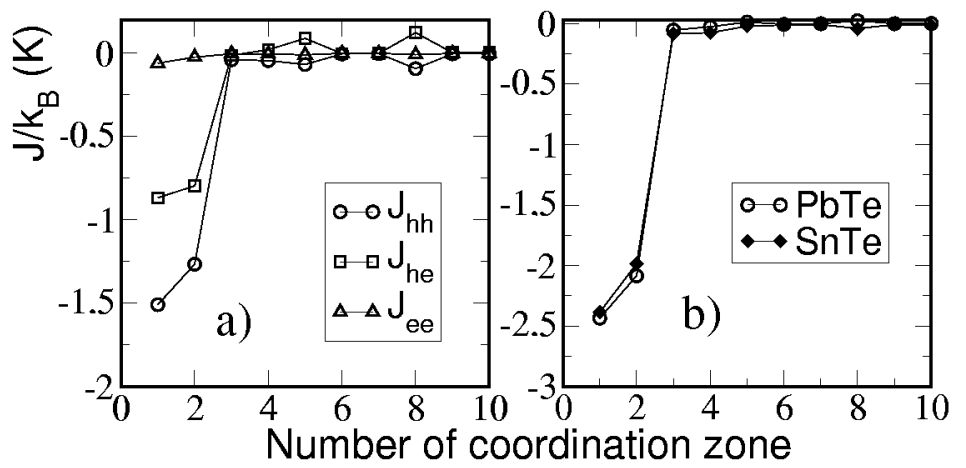

Fig. 1. Contribution of $h \mathrm{~h}, \mathrm{he}$, and ee processes to the exchange integral in PbTe (a). Comparison of the distance dependence of exchange integral in PbTe and $\mathrm{SnTe}$ (b).

Our calculations of the exchange constant, $J(\boldsymbol{R})$, make sense only for $\boldsymbol{R}$ connecting two cation sites in the rocksalt structure lattice. Only for such $\boldsymbol{R}$, i.e. for the case when each of the two $\mathrm{Mn}$ ions is placed in the cation site of the lattice and is surrounded by six Te atoms the hybridization elements $V_{L \sigma, n k}$ are well defined and may be calculated in our model. However, the final formula for the exchange constant is of the form of double integral over the BZ, $\iint \mathrm{d} \boldsymbol{k}_{1} \mathrm{~d} \boldsymbol{k}_{2} \exp \left(\mathrm{i}\left(\boldsymbol{k}_{1}-\boldsymbol{k}_{2}\right) \boldsymbol{R}\right) F\left(\boldsymbol{k}_{1}, \boldsymbol{k}_{2}\right)$, (see Refs. [1] and [13]). The function $F\left(\boldsymbol{k}_{1}, \boldsymbol{k}_{2}\right)$ does not depend on $\boldsymbol{R}$ and we may formally calculate $J(\boldsymbol{R})$ for an arbitrary $\boldsymbol{R}$. We have performed such calculations in order to make comparison with the results presented in the literature, for example in Refs. [1] and [3]. In Fig. 2 we show the dependence of $J(\boldsymbol{R})$ on the Mn-Mn distance for three different directions of $\boldsymbol{R}$. We see that the exchange integral calculated in this way is non-monotonic and 


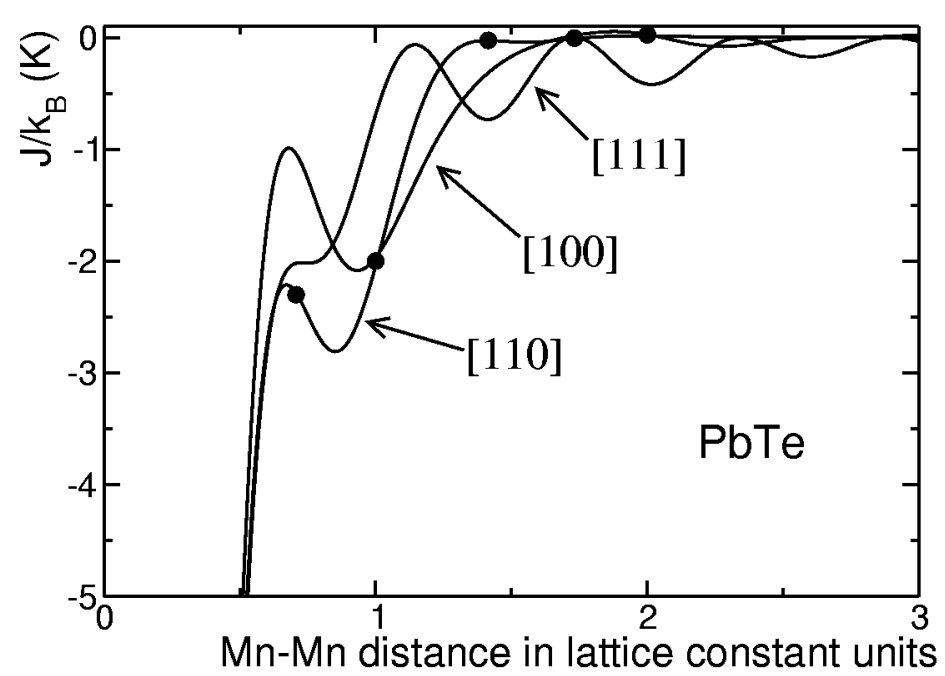

Fig. 2. Dependence of the exchange integral on $\mathrm{Mn}-\mathrm{Mn}$ distance and crystallographic direction. The points on curves correspond to the real cation-cation distance in $\mathrm{NaCl}$ structure (1st, 2nd, 4th, 6th, and 8th nearest neighbour).

strongly anisotropic. This anisotropy is caused mainly by symmetry of the band wave functions, not by the band spin-orbit effects. If in the calculations of the band structure we turn off spin-orbit interactions on $\mathrm{Pb}$ and Te atoms, we obtain practically the same results.

Our results concerning the sign and order of magnitude of the exchange coupling are consistent with the theory and experiment presented in Ref. [4]. Although the absolute values of $J_{\mathrm{NN}}$ obtained in the present paper are about two times larger than those in Ref. [4], by changing the Harrison parameters (which are the main parameters of the model and enter the formulae in the fourth power) by $10-15 \%$ we could have obtained results equal to the experimental ones.

Our results differ from those presented in Refs. [6-8], even up to the sign. We think that the origin of this difference is twofold. First, as we have already discussed, the assumption made in Refs. [6-8] that the most important contributions to the result come from the bands' extrema is not correct, particularly for small Mn-Mn distances. Secondly, as has been pointed out by Larson et al. [1], the assumption of the Kondo-like interaction between band states and the Mn ion rules out some virtual processes which have been taken into account in the present model.

\section{References}

[1] B.E. Larson, K.C. Haas, H. Ehrenreich, A.E. Carlsson, Phys. Rev. B 37, 4137 (1988).

[2] V. Bindilatti, E. ter Haar, N.F. Oliveira, Jr., Y. Shapira, M.T. Liu, Phys. Rev. Lett. 80, 5425 (1998). 
[3] T.M. Rusin, Phys. Rev B 53, 12577 (1996).

[4] M. Górska, J.R. Anderson, Phys. Rev. B 38, 9120 (1988).

[5] P.W. Anderson, in: Magnetism, Eds. G.T. Rado, H. Suhl, Vol. 1, Academic Press, New York 1963, p. 25.

[6] L. Liu, G. Bastard, Phys. Rev. B 25, 487 (1982).

[7] V.K. Dugaev, V.I. Litvinov, Phys. Rev. B 41, 788 (1990).

[8] T.M. Rusin, Phys. Rev. B 53, 7217 (1996).

[9] O. Dimmock, J. Phys. Chem. Solids 32, Suppl. 1, 319 (1971).

[10] M. Kriechbaum, P. Kocevar, H. Pasher, G. Bauer, IEEE J. Quant. Electron. 24, 1727 (1988).

[11] W.A. Harrison, Electronic Structure and the Properties of Solids, Freeman, San Francisco 1980.

[12] J. Mašek, B. Velický, V. Janiš, J. Phys. C: Solid State Phys. 20, 59 (1987).

[13] J. Blinowski, P. Kacman, Acta Phys. Pol. A 87, 539 (1995).

[14] A. Łusakowski, unpublished. 